\title{
2514. A study on the effect of vibration stimulation of the sub-perception threshold intensity on lower leg muscle based on the SEPs
}

\author{
Huigyun Kim ${ }^{1}$, Kiyoung Kwak ${ }^{2}$, Dongwook Kim ${ }^{3}$ \\ ${ }^{1,2}$ Department of Healthcare Engineering, The Graduate School, Chonbuk National University, \\ Jeonju, Republic of Korea \\ ${ }^{3}$ Department of Biomedical Engineering, Chonbuk National University, Jeonju, Republic of Korea \\ ${ }^{3}$ Research Center of Healthcare and Welfare Instrument for Aged, Chonbuk National University, \\ Jeonju, Republic of Korea \\ ${ }^{3}$ Corresponding author \\ E-mail: ${ }^{1}$ hekun1249@naver.com, ${ }^{2} k y k w a k 86 @ n a t e . c o m,{ }^{3}$ biomed@jbnu.ac.kr
}

Received 11 October 2016; received in revised form 8 April 2017; accepted 12 April 2017

DOI https://doi.org/10.21595/jve.2017.18369

\begin{abstract}
This paper aims to study the effect of vibration stimulation of the sub-perception threshold on the tibialis anterior muscle and triceps surae muscle based on the somatosensory evoked potentials (SEPs). EEG is measured by applying the vibration stimulation to tibialis anterior tendon and Achilles tendon, and the sub-perception threshold intensity is selected through SEPs analysis. For this purpose, EEC collection and analysis system was used. In addition, analyzing changes in muscle strength and muscle reaction time by applying the selected sub-perception threshold intensity to tibialis anterior tendon and Achilles tendon. For this, maximum voluntary contraction (MVC), and muscle reaction time (MRT) are analyzed by using the EMG collection and analysis system. Vibration stimulation of the sub-perception threshold intensity analysis results, muscle strength is increased, muscle reaction time decreased.
\end{abstract}

Keywords: sub-perception threshold, vibration stimulation, somatosensory evoked potentials (SEPs), maximum voluntary contraction (MVC), muscle reaction time (MRT).

\section{Introduction}

The postural stability is the ability to control the balance of the human body in the support surface while maintaining posture [1]. The postural control needs various kinds of sensory information and motions and their interactions such as vision, sense of equilibrium, muscle spindle, skin, muscle and receptor of tendon [2]. Muscle strength shows high correlation with postural stability [3]. But the reduction in muscle strength due to aging has a negative effect on postural stability and increases the possibility of abnormal gait and falls [4]. For enhancement in postural stability, there is a need to improve the sensory and motor abilities necessary to postural control.

This study aims to find out the effect the vibration stimulation introduced to the somatosensory system among many senses required for the postural control through the small-linear actuator has on the improvement of postural stability. The stimulation to the somatosensory system is to activate the tactile sense, pressure sense, vibration sense and the receptor of position and motor sense to stimulate the sensory stimulation route connected to the cerebrum [5]. There are various studies showing that the somatosensory stimulation reduces the body sway and enhances the postural stability in static posture [6,7]. However, there are problems in the safety of the research using the electric stimulation. Some research reported that there was body sway even with somatosensory stimulation using a small vibrator having less problems in stability [8] and that even minute vibration affects the human's sense of balance [9]. There are many researches conducted on not only the relation between somatosensory system and postural balance but also on the various body responses caused by somatosensory stimulation $[10,11]$.

However, existing studies have limitations in that they did not consider the individual differences in terms of vibration frequency and intensity of each vibration though they have proven 
the effect of the vibration stimulation on the improvement of postural stability. Therefore, in this study, the perception threshold value was measured and applied to overcome the limitations above based on the preceding researches on the band of frequency in which the somatosensory receptor sensitively responds as it is the value in which an individual can sense vibration [12]. In addition, the stimulation having the sub-perception threshold intensity which a person cannot feel but can affect the human body was investigated through analyzing Somatosensory Evoked Potentials (SEPs). Based on the results from analyzing SEPs, the effect of the vibration stimulation applied to the somatosensory system depending on the conditions of vibration stimulation (stimulation site, frequency and intensity) on the postural stability was interpreted through the Maximum Voluntary Contraction (MVC) and Muscle Reaction Time (MRT). The change in muscle strength depending on the vibration stimulation has been studied using MVC as MVC means the maximum power which can be produced by the group of muscles through maximum voluntary control [13]. Using the response time which means the time from the time when stimulation is given to the time when the response is shown [14], we have studied the changes in the muscle reaction time depending on the vibration stimulation.

\section{Methods}

\subsection{Subjects}

This study was conducted targeting male adults having no neuropathy or musculoskeletal diseases. 7 subjects participated (Age: $27.1 \pm 1.2$, Hight: $173.5 \mathrm{~cm} \pm 2.2$, Weight: $68.1 \mathrm{~kg} \pm 4.2$ ) in the SEPs experiment. 10 subjects participated (Age: $26.9 \pm 1.5$, Height: $171.2 \mathrm{~cm} \pm 2.5$, Weight: $65.9 \mathrm{~kg} \pm 5.3$ ) in the experiment for measuring MVC and MRT. Subjects did not participate in similar experiments and did not experience vibration exercise. The study was approved by the IRB of Chonbuk National University (IRB File No. JBNU 2015-06-012).

\subsection{Equipment}

The study was conducted in a dark room to block out the light and noise from the outside. The small-linear actuator (DMJBRN0934AA, SAMSUNG ELECTRO-MECHANICS Co., Ltd., Korea) was used to introduce the vibration stimulation. The function generator (AFG-2125, GOOD WILL INSTRUMENT Co., Ltd., Taiwan) was used to adjust the vibration frequency and intensity. The rubber band was used to fix the small-linear actuator to the vibration stimulation area (tibialis anterior tendon and Achilles tendon).

The EEG collection program (Brain-Products $\mathrm{GmbH}$, Germany) and EEG analysis program (BESA GmbH, Germany) were used to check the changes in the EEG depending on the application of vibration stimulation. For the collection of EEG, three ranges of the disk electrodes $(\mathrm{C} 3, \mathrm{Cz}$ and $\mathrm{C} 4$ ) associated with the somatosensory area were used according to the internationally standardized 10-20 system [15]. EEG was synchronized to the trigger signal to which the vibration stimulation is applied.

EMG measuring system (Bagnoli ${ }^{\mathrm{TM}}$ Desktop EMG Systems, DELSYS Inc, USA) was used to check the changes in EMG depending on the application of vibration stimulation. For the collection of EMG, the electrodes were attached to the tibialis anterior muscle and triceps surae muscle. The ground electrode was attached to the patella. The audio signal was composed of the MRT test and EMG was synchronized to the trigger signal where the vibration stimulation and audio signal start.

\subsection{Protocol}

To study the changes in EEG and EMG depending on the vibration stimulation, the vibration perception threshold was measured on the tibialis anterior tendon and Achilles tendon through the 
vibration frequency $(180 \mathrm{~Hz}, 190 \mathrm{~Hz}, 250 \mathrm{~Hz})$ which is selected based on precedent studies on the perception threshold depending on the vibration frequency [12]. The vibration stimulation conditions were made by combining the stimulation site (tibialis anterior tendon, Achilles tendon), vibration frequency, the perception threshold intensity $(100 \%)$ where the vibration starts to be felt and sub-perception threshold intensity (70-95\%). As an example of vibration stimulation conditions, there is "the $180 \mathrm{~Hz}$ perception threshold intensity stimulation was applied to the Achilles tendon".

\subsubsection{Protocol of somatosensory evoked potentials}

For the collection of EEG, a subject was asked to comfortably sit down on a seat and the disk electrode was attached to the scalp. 100 vibration stimulation were applied to the subject under the vibration stimulation conditions with the interval of 0.5 second between stimulation. At this time, EEG was collected. Once each vibration stimulation condition cycle was completed, the subject was given a 3 minutes break. The vibration stimulation condition was applied in a random manner.

\subsubsection{Protocol of maximum voluntary contraction and muscle reaction time}

For the collection of EMG data, the subject was tested 3 times each with MVC and MRT under each condition. At this time, the EMG was collected. In case of MVC, the subject performed muscle contraction with maximum strength at the same time the vibration was applied. In case of MRT, the subject performed muscle contraction as fast as one can at the same time as the application of the audio signal. The time from the starting time of audio signal (when EMG measuring starts) to the time when EMG is activated was obtained. Once each vibration stimulation condition cycle was completed, a 3 minutes break was given to the subject. The vibration stimulation condition was applied randomly.

\subsection{Analysis}

\subsubsection{Analysis of somatosensory evoked potentials}

EEG was collected in 3 areas using the EEG collection program. The sampling rate was set to $1000 \mathrm{~Hz}$. To remove noise in the measuring of brain wave, High pass filter $-0.1 \mathrm{~Hz}$, Low pass filter $-70 \mathrm{~Hz}$, and Notch filter $-60 \mathrm{~Hz}$ were applied. The amplitude of SEPs was analyzed as they sensed the electric activities in the brain which occur at a certain time related to the vibratory somatosensory stimulation in EEG data. The amplitude of SEPs at the 100-150 ms (N150) and 220-350 ms (P260) was obtained based on the application start marker of the vibration stimulation [16]. N150 means, the negative peak in the range of $100-150 \mathrm{~ms}$ based on the application start marker of the vibration stimulation. P260 means, the positive peak in the range of 220-350 ms based on the application start marker of the vibration stimulation. The change in the somatosensory stimulation through vibration was interpreted by comparing the amplitude of SEPs between when there is vibration stimulation and when there is no vibration stimulation.

\subsubsection{Analysis of maximum voluntary contraction and pre-motor time}

EMG from anterior tibial muscle and triceps surae muscle was obtained using EMG collection program. In case of MVC, the peak value of RMS (root-means square) EMG was derived from the EMG data to analyze the change in muscle strength. In case of MRT, the duration starting on the time of application of audio signal and ending on the time of EMG activation was obtained to analyze the change in muscle reaction time. The change in muscle strength and the muscle reaction time was interpreted by comparing the increase or decrease between the EMG data obtained under the stimulation condition and those under the non-stimulation condition. 
The paired $t$-test was conducted using SPSS 18.0 (IBM Co., Ltd. USA) with significance level of $p<0.05$.

\section{Results and discussion}

\subsection{Change in the amplitude in the somatosensory evoked potential depending on the conditions of vibration stimulation}

Table 1 shows that when the stimulation is applied to the tibialis anterior tendon according to the vibration condition, the amplitude of SEPs (N150 component) tended to increase more than that in the non-stimulation as it gets closer to the perception threshold. The amplitude from the sub-perception threshold ( $80 \%$ intensity) shows a more significant increase than that in non-stimulation amplitude $\left(180 \mathrm{~Hz} \_\mathrm{C} 3=80-100 \%, 180 \mathrm{~Hz} \_\mathrm{Cz}=90-100 \%, 190 \mathrm{~Hz} \_\mathrm{C} 3=80-\right.$ $\left.100 \%, 190 \mathrm{~Hz} \_\mathrm{Cz}=90-100 \%, 190 \mathrm{~Hz} \_\mathrm{C} 4=95-100 \%\right)$.

Table 1. Change in the amplitude of SEPs-N150 depending on the vibration stimulation applied through the Tibialis Anterior Tendon

\begin{tabular}{|c|c|c|c|c|c|c|c|c|c|c|}
\hline & \multicolumn{8}{|c|}{ SEPs amplitude $(\mu \mathrm{V})$} \\
\hline & & & None & $70 \%$ & $75 \%$ & $80 \%$ & $85 \%$ & $90 \%$ & $95 \%$ & $100 \%$ \\
\hline \multirow{18}{*}{$\begin{array}{l}\text { Tibialis } \\
\text { Anterior } \\
\text { Tendon } \\
(\mathrm{N} 150)\end{array}$} & \multirow{6}{*}{$180 \mathrm{~Hz}$} & \multirow{2}{*}{ C3 } & $-3.12 *$ & -3.24 & -4.38 & $-4.67 *$ & $-5.38 *$ & $-5.32 *$ & $-5.14 *$ & $-5.77 *$ \\
\hline & & & \pm 1.24 & \pm 2.52 & \pm 2.99 & \pm 1.04 & \pm 0.82 & \pm 1.25 & \pm 0.98 & \pm 1.36 \\
\hline & & \multirow{2}{*}{$\mathrm{Cz}$} & $-3.78^{*}$ & -3.69 & -3.71 & -3.99 & -4.14 & $-5.23 *$ & $-4.98^{*}$ & $-5.43 *$ \\
\hline & & & \pm 1.03 & \pm 1.49 & \pm 1.57 & \pm 2.07 & \pm 1.84 & \pm 0.93 & \pm 0.51 & \pm 1.02 \\
\hline & & \multirow{2}{*}{$\mathrm{C} 4$} & -4.23 & -4.11 & -4.02 & -3.86 & -3.35 & -4.03 & -3.77 & -3.98 \\
\hline & & & \pm 1.57 & \pm 1.66 & \pm 2.14 & \pm 1.85 & \pm 2.48 & \pm 2.35 & \pm 1.96 & \pm 2.08 \\
\hline & \multirow{6}{*}{$190 \mathrm{~Hz}$} & \multirow{2}{*}{ C3 } & $-3.12^{*}$ & -3.06 & -3.31 & $-4.74 *$ & $-5.42 *$ & $-6.82 *$ & $-6.55^{*}$ & $-6.89 *$ \\
\hline & & & \pm 1.24 & \pm 1.08 & \pm 1.54 & \pm 0.36 & \pm 0.97 & \pm 1.34 & \pm 1.22 & \pm 1.68 \\
\hline & & \multirow{2}{*}{$\mathrm{Cz}$} & $-3.78^{*}$ & -3.99 & -3.52 & -4.12 & -4.99 & $-5.44 *$ & -5.19 & $-5.25^{*}$ \\
\hline & & & \pm 1.03 & \pm 1.32 & \pm 1.01 & \pm 1.85 & \pm 2.06 & \pm 1.22 & \pm 3.36 & \pm 1.11 \\
\hline & & \multirow{2}{*}{ C4 } & $-4.23 *$ & -3.89 & -4.11 & -4.92 & -5.58 & -5.24 & $-5.43^{*}$ & $-5.62 *$ \\
\hline & & & \pm 1.57 & \pm 1.53 & \pm 1.41 & \pm 1.33 & \pm 3.07 & \pm 3.01 & \pm 1.24 & \pm 0.98 \\
\hline & \multirow{6}{*}{$250 \mathrm{~Hz}$} & \multirow{2}{*}{ C3 } & -3.12 & -3.31 & -2.56 & -3.54 & -4.18 & -3.04 & -2.14 & -2.43 \\
\hline & & & \pm 1.24 & \pm 1.98 & \pm 2.22 & \pm 2.67 & \pm 2.95 & \pm 1.10 & \pm 1.77 & \pm 2.46 \\
\hline & & \multirow{2}{*}{$\mathrm{Cz}$} & -3.78 & -3.75 & -3.62 & -3.81 & -4.27 & -4.23 & -4.17 & -4.22 \\
\hline & & & \pm 1.03 & \pm 1.88 & \pm 1.99 & \pm 2.28 & \pm 3.02 & \pm 2.99 & \pm 4.03 & \pm 5.21 \\
\hline & & \multirow{2}{*}{$\mathrm{C} 4$} & -4.23 & -4.11 & -4.89 & -3.65 & -3.25 & -4.12 & -4.26 & -3.52 \\
\hline & & & \pm 1.57 & \pm 2.84 & \pm 1.39 & \pm 2.04 & \pm 2.69 & \pm 4.03 & \pm 3.41 & \pm 2.55 \\
\hline
\end{tabular}

Table 2 shows that when the stimulation is applied to the tibialis anterior tendon according to the vibration condition, the amplitude of SEPs (P260 component) tended to increase more than that in the non-stimulation as it gets closer to the perception threshold. The amplitude from the sub-perception threshold ( $80 \%$ intensity) shows a more significant increase than that in nonstimulation amplitude $\left(180 \mathrm{~Hz} \mathrm{Cz}_{2}=85.95 \cdot 100 \%, 180 \mathrm{~Hz} \_\mathrm{C} 4=80-100 \%, 190 \mathrm{~Hz} \mathrm{C} 3=90 \%\right.$, $\left.190 \mathrm{~Hz} \_\mathrm{Cz}=100 \%, 190 \mathrm{~Hz} \_\mathrm{C} 4=85-100 \%, 250 \mathrm{~Hz} \mathrm{C} 3=80-90 \%, 250 \mathrm{~Hz} \_\mathrm{C} 4=95 \%\right)$.

Table 3 shows that when the stimulation is applied to the Achilles tendon according to the vibration condition, the amplitude of SEPs (N150 component) tended to increase more than that in the non-stimulation as it gets closer to the perception threshold. The amplitude from the subperception threshold (80\% intensity) shows a more significant increase than that in nonstimulation amplitude $\left(180 \mathrm{~Hz} \_\mathrm{C} 3=85 \cdot 95 \cdot 100 \%, 180 \mathrm{~Hz}-\mathrm{Cz}=90-100 \%, 190 \mathrm{~Hz} \mathrm{C} 3=90-\right.$ $100 \%, 190 \mathrm{~Hz} \_\mathrm{Cz}=90-100 \%, 190 \mathrm{~Hz} \_\mathrm{C} 4=100 \%, 250 \mathrm{~Hz} \_\mathrm{C} 3=85 \cdot 95 \cdot 100 \%, 250 \mathrm{~Hz} \_\mathrm{Cz}=$ 
$\left.85 \cdot 95 \cdot 100 \%, 250 \mathrm{~Hz} \_\mathrm{C} 4=80 \cdot 95 \cdot 100 \%\right)$.

Table 2. Change in the amplitude of SEPs-P260 depending on the vibration stimulation applied through the Tibialis Anterior Tendon

\begin{tabular}{|c|c|c|c|c|c|c|c|c|c|c|}
\hline & \multicolumn{8}{|c|}{ SEPs amplitude $(\mu \mathrm{V})$} \\
\hline & & & None & $70 \%$ & $75 \%$ & $80 \%$ & $85 \%$ & $90 \%$ & $95 \%$ & $100 \%$ \\
\hline \multirow{18}{*}{$\begin{array}{l}\text { Tibialis } \\
\text { Anterior } \\
\text { Tendon } \\
\text { (P260) }\end{array}$} & \multirow{6}{*}{$180 \mathrm{~Hz}$} & \multirow{2}{*}{$\mathrm{C} 3$} & 3.32 & 3.14 & 3.42 & 3.66 & 3.37 & 4.33 & 4.01 & 4.12 \\
\hline & & & \pm 1.18 & \pm 1.21 & \pm 1.64 & \pm 1.74 & \pm 1.36 & \pm 2.12 & \pm .1 .63 & \pm 3.12 \\
\hline & & \multirow{2}{*}{$\mathrm{Cz}$} & $3.78 *$ & 3.57 & 3.72 & 4.18 & $5.36^{*}$ & 5.14 & $5.27 *$ & $5.56^{*}$ \\
\hline & & & \pm 1.62 & \pm 0.86 & \pm 2.31 & \pm 1.10 & \pm 0.76 & \pm 1.80 & \pm 0.68 & \pm 1.06 \\
\hline & & \multirow{2}{*}{$\mathrm{C} 4$} & $4.13 *$ & 3.67 & 3.92 & $5.63^{*}$ & $6.27 *$ & $5.76^{*}$ & $6.23 *$ & $6.18 *$ \\
\hline & & & \pm 1.96 & \pm 0.89 & \pm 0.87 & \pm 1.01 & \pm 0.54 & \pm 0.21 & \pm 1.26 & \pm 1.18 \\
\hline & \multirow{6}{*}{$190 \mathrm{~Hz}$} & \multirow{2}{*}{$\mathrm{C} 3$} & $3.32 *$ & 3.77 & 3.68 & 4.16 & 4.09 & $5.14 *$ & 4.57 & 4.55 \\
\hline & & & \pm 1.18 & \pm 1.25 & \pm 0.99 & \pm 1.25 & \pm 1.79 & \pm 1.01 & \pm 2.34 & \pm 2.53 \\
\hline & & \multirow{2}{*}{$\mathrm{Cz}$} & $3.78 *$ & 3.67 & 3.87 & 4.12 & 4.19 & 5.02 & 4.92 & $5.13 *$ \\
\hline & & & \pm 1.62 & \pm 1.44 & \pm 1.73 & \pm 1.73 & \pm 1.99 & \pm 2.36 & \pm 2.89 & \pm 0.55 \\
\hline & & \multirow{2}{*}{$\mathrm{C} 4$} & $4.13 *$ & 3.89 & 4.05 & 5.18 & $5.77 *$ & $6.31 *$ & $7.23 *$ & $6.34^{*}$ \\
\hline & & & \pm 1.96 & \pm 1.56 & \pm 1.22 & \pm 2.12 & \pm 0.71 & \pm 0.58 & \pm 1.24 & \pm 1.21 \\
\hline & \multirow{6}{*}{$250 \mathrm{~Hz}$} & \multirow{2}{*}{ C3 } & $3.32 *$ & 3.38 & 3.76 & $4.92 *$ & $5.34 *$ & $5.02 *$ & 4.31 & 4.11 \\
\hline & & & \pm 1.18 & \pm 1.74 & \pm 1.44 & \pm 0.31 & \pm 0.34 & \pm 0.12 & \pm 2.21 & \pm 3.25 \\
\hline & & \multirow{2}{*}{$\mathrm{Cz}$} & 3.78 & 3.45 & 4.15 & 4.42 & 3.24 & 3.51 & 4.15 & 3.26 \\
\hline & & & \pm 1.62 & \pm 0.99 & \pm 1.54 & \pm 2.65 & \pm 1.89 & \pm 2.12 & \pm 2.26 & \pm 4.13 \\
\hline & & \multirow{2}{*}{$\mathrm{C} 4$} & $4.13^{*}$ & 3.89 & 4.02 & 4.52 & 5.01 & 4.77 & $5.23 *$ & 4.98 \\
\hline & & & \pm 1.96 & \pm 1.16 & \pm 2.15 & \pm 2.98 & \pm 3.24 & \pm 1.87 & \pm 0.88 & \pm 2.27 \\
\hline
\end{tabular}

Table 3. Change in the amplitude of SEPs-N150 depending on the vibration stimulation applied through the Achilles Tendon

\begin{tabular}{|c|c|c|c|c|c|c|c|c|c|c|}
\hline & \multicolumn{8}{|c|}{ SEPs amplitude $(\mu V)$} \\
\hline & & & None & $70 \%$ & $75 \%$ & $80 \%$ & $85 \%$ & $90 \%$ & $95 \%$ & $100 \%$ \\
\hline \multirow{18}{*}{$\begin{array}{l}\text { Achilles } \\
\text { Tendon } \\
\text { (N150) }\end{array}$} & \multirow{6}{*}{$180 \mathrm{~Hz}$} & \multirow{2}{*}{$\mathrm{C} 3$} & $-3.12 *$ & -3.27 & -3.76 & -4.23 & $-5.33 *$ & -5.18 & $-5.21 *$ & $-5.23 *$ \\
\hline & & & \pm 1.24 & \pm 1.55 & \pm 1.23 & \pm 1.71 & \pm 1.42 & \pm 3.45 & \pm 0.98 & \pm 1.21 \\
\hline & & \multirow{2}{*}{$\mathrm{Cz}$} & $-3.78 *$ & -3.54 & -3.92 & -4.22 & -7.01 & $-5.87 *$ & $-6.01 *$ & $-6.18 *$ \\
\hline & & & \pm 1.03 & \pm 2.12 & \pm 1.24 & \pm 1.21 & \pm 4.23 & \pm 1.29 & \pm 1.34 & \pm 2.01 \\
\hline & & \multirow{2}{*}{$\mathrm{C} 4$} & -4.23 & -3.21 & -4.76 & -5.21 & -6.23 & -4.77 & -5.89 & -4.61 \\
\hline & & & \pm 1.57 & \pm 3.21 & \pm 1.25 & \pm 3.21 & \pm 2.33 & \pm 1.23 & \pm 3.56 & \pm 3.12 \\
\hline & \multirow{6}{*}{$190 \mathrm{~Hz}$} & \multirow{2}{*}{ C3 } & $-3.12 *$ & -3.36 & -3.78 & -4.22 & -4.23 & $-5.24 *$ & $-5.57 *$ & $-5.89 *$ \\
\hline & & & \pm 1.24 & \pm 1.22 & \pm 0.84 & \pm 2.09 & \pm 2.32 & \pm 0.54 & \pm 0.67 & \pm 1.08 \\
\hline & & \multirow{2}{*}{$\mathrm{Cz}$} & $-3.78^{*}$ & -3.67 & -3.55 & -3.91 & -4.52 & $-6.23 *$ & $-5.89 *$ & $-6.42 *$ \\
\hline & & & \pm 1.03 & \pm 1.71 & \pm 0.45 & \pm 1.11 & \pm 2.76 & \pm 1.21 & \pm 1.04 & \pm 1.34 \\
\hline & & \multirow{2}{*}{$\mathrm{C} 4$} & $-4.23 *$ & -4.12 & -4.28 & -5.15 & -4.95 & -3.23 & -5.42 & $-5.77 *$ \\
\hline & & & \pm 1.57 & \pm 1.80 & \pm 1.04 & \pm 2.67 & \pm 2.01 & \pm 3.45 & \pm 2.02 & \pm 1.23 \\
\hline & \multirow{6}{*}{$250 \mathrm{~Hz}$} & \multirow{2}{*}{ C3 } & $-3.12 *$ & -2.89 & -3.06 & -3.19 & $-5.23 *$ & -5.04 & $-5.17 *$ & $-5.11 *$ \\
\hline & & & \pm 1.24 & \pm 1.87 & \pm 1.90 & \pm 2.26 & \pm 0.90 & \pm 2.92 & \pm 0.21 & \pm 0.87 \\
\hline & & \multirow{2}{*}{$\mathrm{Cz}$} & $-3.78^{*}$ & -4.89 & -5.27 & -5.12 & $-5.77^{*}$ & -5.32 & $-5.77^{*}$ & $-5.57 *$ \\
\hline & & & \pm 1.03 & \pm 2.04 & \pm 2.46 & \pm 2.35 & \pm 1.27 & \pm 2.33 & \pm 1.33 & \pm 0.24 \\
\hline & & \multirow{2}{*}{$\mathrm{C} 4$} & $-4.23 *$ & -5.03 & -4.12 & $-6.33^{*}$ & -8.76 & -5.45 & $-6.24 *$ & $-6.59 *$ \\
\hline & & & \pm 1.57 & \pm 2.02 & \pm 1.78 & \pm 1.29 & \pm 5.08 & \pm 2.87 & \pm 1.04 & \pm 1.17 \\
\hline
\end{tabular}


Table 4 shows that when the stimulation is applied to the Achilles tendon according to the vibration condition, the amplitude of SEPs (P260 component) tended to increase more than that in the non-stimulation as it gets closer to the perception threshold. The amplitude from the subperception threshold (80\% intensity) shows a more significant increase than that in nonstimulation amplitude $(180 \mathrm{~Hz} C 3=80.90 \%, 180 \mathrm{~Hz} \mathrm{Cz}=90 \%, 180 \mathrm{~Hz} \mathrm{C} 4=95 \%$, $190 \mathrm{~Hz} \_\mathrm{C} 3=80 \cdot 95 \cdot 100 \%, \quad 190 \mathrm{~Hz} \mathrm{Cz}_{-}=80 \cdot 90 \cdot 100 \%, 190 \mathrm{~Hz}{ }_{-} \mathrm{C} 4=90-100 \%$, $250 \mathrm{~Hz} \_\mathrm{C} 3=85 \%, 250 \mathrm{~Hz} \_\mathrm{C} 4=90-100 \%$ ).

Table 4. Change in the amplitude of SEPs-P260 depending on the vibration stimulation applied through the Achilles Tendon

\begin{tabular}{|c|c|c|c|c|c|c|c|c|c|c|}
\hline & \multicolumn{8}{|c|}{ SEPs amplitude $(\mu V)$} \\
\hline & & & None & $70 \%$ & $75 \%$ & $80 \%$ & $85 \%$ & $90 \%$ & $95 \%$ & $100 \%$ \\
\hline \multirow{18}{*}{$\begin{array}{l}\text { Achilles } \\
\text { Tendon } \\
\text { (P260) }\end{array}$} & \multirow{6}{*}{$180 \mathrm{~Hz}$} & \multirow{2}{*}{$\mathrm{C} 3$} & $3.32 *$ & 3.79 & 4.33 & $5.07 *$ & 4.78 & $4.99 *$ & 4.37 & 4.14 \\
\hline & & & \pm 1.18 & \pm 1.43 & \pm 1.67 & \pm 0.23 & \pm 1.95 & \pm 0.11 & \pm 2.55 & \pm 2.33 \\
\hline & & \multirow{2}{*}{$\mathrm{Cz}$} & $3.78^{*}$ & 3.92 & 3.18 & 4.36 & 4.77 & $5.14^{*}$ & 5.07 & 4.89 \\
\hline & & & \pm 1.62 & \pm 1.22 & \pm 1.25 & \pm 2.32 & \pm 1.33 & \pm 0.27 & \pm 3.21 & \pm 3.56 \\
\hline & & \multirow{2}{*}{$\mathrm{C} 4$} & $4.13^{*}$ & 4.19 & 5.06 & 4.89 & 6.53 & 8.23 & $6.88^{*}$ & $7.02 *$ \\
\hline & & & \pm 1.96 & \pm 1.14 & \pm 2.56 & \pm 4.12 & \pm 3.52 & \pm 5.67 & \pm 2.11 & \pm 2.21 \\
\hline & \multirow{6}{*}{$190 \mathrm{~Hz}$} & \multirow{2}{*}{$\mathrm{C} 3$} & $3.32 *$ & 3.55 & 3.76 & $5.23 *$ & 4.12 & 4.56 & $4.81 *$ & $4.88 *$ \\
\hline & & & \pm 1.18 & \pm 1.89 & \pm 2.01 & \pm 1.01 & \pm 2.23 & \pm 2.35 & \pm 0.98 & \pm 1.22 \\
\hline & & \multirow{2}{*}{$\mathrm{Cz}$} & $3.78^{*}$ & 3.12 & 3.89 & $7.25 *$ & 6.12 & $5.66^{*}$ & 5.23 & $5.74 *$ \\
\hline & & & \pm 1.62 & \pm 1.98 & \pm 2.33 & \pm 2.35 & \pm 4.52 & \pm 0.36 & \pm 3.04 & \pm 1.01 \\
\hline & & \multirow{2}{*}{$\mathrm{C} 4$} & $4.13^{*}$ & 4.32 & 3.78 & 5.09 & 5.26 & $6.14 *$ & $6.05 *$ & $6.02 *$ \\
\hline & & & \pm 1.96 & \pm 1.56 & \pm 2.72 & \pm 4.17 & \pm 3.75 & \pm 1.00 & \pm 0.74 & \pm 0.33 \\
\hline & \multirow{6}{*}{$250 \mathrm{~Hz}$} & \multirow{2}{*}{$\mathrm{C} 3$} & $3.32 *$ & 3.56 & 3.12 & 3.38 & $5.17^{*}$ & 3.31 & 3.16 & 3.05 \\
\hline & & & \pm 1.18 & \pm 2.33 & \pm 3.02 & \pm 2.99 & \pm 0.91 & \pm 2.62 & \pm 1.20 & \pm 2.33 \\
\hline & & \multirow{2}{*}{$\mathrm{Cz}$} & 3.78 & 2.98 & 3.12 & 3.24 & 4.02 & 3.96 & 4.18 & 4.12 \\
\hline & & & \pm 1.62 & \pm 3.23 & \pm 1.24 & \pm 2.45 & \pm 1.33 & \pm 1.77 & \pm 2.23 & \pm 4.23 \\
\hline & & \multirow{2}{*}{$\mathrm{C} 4$} & $4.13 *$ & 4.29 & 4.14 & 3.89 & 4.55 & $6.23 *$ & $5.55 *$ & $5.81 *$ \\
\hline & & & \pm 1.96 & \pm 2.08 & \pm 1.66 & \pm 2.36 & \pm 2.34 & \pm 1.02 & \pm 0.44 & \pm 1.15 \\
\hline
\end{tabular}

Tables 1-4 show that when the vibration stimulation is applied to the tibialis anterior tendon and Achilles tendon, the amplitude of SEPs increases more than that in the case of non-vibration. This means that the vibration stimulation evoked potential stimulates somatosensory receptors occurs. As for the perception threshold stimulation depending on the vibration frequency, the amplitude shows a different trend depending on the frequency. This applies to the stimulation the sub-perception threshold intensity. This shows that the vibration frequency has an effect on the change in amplitude. In addition, if the subject has applied the sub-perception threshold ( $80 \%$ intensity) of the vibration stimulation that does not feel the vibration, the amplitude of SEPs increases more than in the case of non-vibration. This means that stimulation of sub-perception threshold intensity to generate evoked potential, which means that it is possible to influence the vibration to the human body. It can be used for the research on the improvement in postural stabilization. Based on the results from the research on the change in amplitude of the SEPs, $80 \%$ of the value below the perception threshold was applied for MVC and MRT experiments.

\subsection{Change in muscle strength of MVC depending on the conditions of vibration stimulation}

Change in muscle strength of MVC depending on the conditions of vibration stimulation was analyzed based on MVC data under the conditions of non-stimulation.

Fig. 1 showed that muscle strength increased more in vibration stimulation than in non- 
stimulation. When the vibration stimulation is applied to tibialis anterior tendon, muscle strength in the medial gastrocnemius muscle had a more significant increase than that under non-stimulation (Medial Gastrocnemius $p$-value $=0.017$ ).

When the vibration stimulation is applied to the Achilles tendon, muscle strength in the soleus muscle, medial gastrocnemius muscle, lateral gastrocnemius muscle, and tibialis anterior muscle had a more significant increase than that under non-stimulation (Soleus $p$-value $=0.022$, Lateral Gastrocnemius $p$-value $=0.049$, Medial Gastrocnemius $p$-value $=0.033$, Tibialis Anterior $p$-value $=0.047)$.

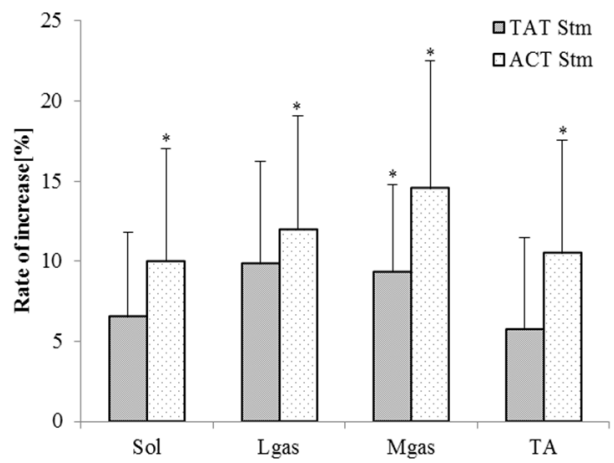

Fig. 1. Muscle strength increasing rate [\%] of MVC by the applied vibration stimulation to the tibialis anterior and Achilles tendon. TAT Stm: Tibialis Anterior Tendon Stimulation, ACT Stm: Achilles Tendon Stimulation, Sol: Soleus, Lgas: Lateral Gastrocnemius, Mgas: Medial Gastrocnemius, TA: Tibialis Anterior. ${ }^{*}: p<0.05$. Non-Stimulation versus Stimulation

Table 5 When vibration stimulation was applied to tibialis anterior tendon, the rates of increase in muscle strength of soleus muscle and tibialis anterior muscle were the highest in $180 \mathrm{~Hz}$ and the lowest in $250 \mathrm{~Hz}$, while the rates of increase in muscle strength of lateral gastrocnemius muscle and medial gastrocnemius muscle were the highest in $180 \mathrm{~Hz}$ and the lowest in $190 \mathrm{~Hz}$ (Lateral Gastrocnemius: $180 \mathrm{~Hz}-190 \mathrm{~Hz} p$-value $=0.007$, Medial Gastrocnemius: $180 \mathrm{~Hz}-190 \mathrm{~Hz} p$-value $=0.019)$.

When the vibration stimulation was applied to Achilles tendon, the rates of increase in muscle strength of soleus muscle and tibialis anterior muscle were the highest in $180 \mathrm{~Hz}$ and the lowest in $190 \mathrm{~Hz}$, while the rates of increase in muscle strength of lateral gastrocnemius muscle and medial gastrocnemius muscle were the highest in $180 \mathrm{~Hz}$ and the lowest in $250 \mathrm{~Hz}$.

Table 5. Muscle strength increasing rate [\%] of MVC in accordance with the vibration frequency applied to the tibialis anterior and Achilles tendon

\begin{tabular}{|c|c|c|c|c|c|}
\hline \multicolumn{2}{|c|}{} & Soleus & $\begin{array}{c}\text { Lateral } \\
\text { Gastrocnemius }\end{array}$ & $\begin{array}{c}\text { Medial } \\
\text { Gastrocnemius }\end{array}$ & $\begin{array}{c}\text { Tibialis } \\
\text { Anterior }\end{array}$ \\
\hline \multirow{2}{*}{$\begin{array}{c}\text { Tibialis } \\
\text { Anterior } \\
\text { Tendon }\end{array}$} & $180 \mathrm{~Hz}$ & $9.34 \pm 3.57$ & $13.81 \pm 6.02^{*}$ & $13.04 \pm 6.11^{*}$ & $7.39 \pm 6.61$ \\
\cline { 2 - 6 } & $190 \mathrm{~Hz}$ & $5.97 \pm 5.92$ & $6.46 \pm 6.53^{*}$ & $6.41 \pm 5.02^{*}$ & $5.13 \pm 6.37$ \\
\hline \multirow{2}{*}{$\begin{array}{c}\text { Achilles } \\
\text { Tendon }\end{array}$} & $180 \mathrm{~Hz}$ & $4.29 \pm 6.24$ & $9.21 \pm 6.84$ & $8.57 \pm 5.06$ & $4.69 \pm 4.59$ \\
\cline { 2 - 6 } & $190 \mathrm{~Hz}$ & $11.52 \pm 8.02$ & $14.54 \pm 7.71$ & $16.54 \pm 8.13$ & $11.30 \pm 7.15$ \\
\cline { 2 - 6 } & $250 \mathrm{~Hz}$ & $11.52 \pm 4.19$ & $12.52 \pm 6.22$ & $14.50 \pm 9.16$ & $8.57 \pm 9.02$ \\
\hline \multirow{2}{*}{$*$}
\end{tabular}

$*: P<0.05 .180 \mathrm{~Hz}$ versus $190 \mathrm{~Hz}$ of Lateral Gastrocnemius and Medial Gastrocnemius

Table 6 When the vibration stimulation was applied to tibialis anterior tendon, there was a difference in the increase in muscle strength among soleus muscle, lateral gastrocnemius muscle, medial gastrocnemius muscle and tibialis anterior muscle depending on the perception threshold intensity and the sub-perception threshold intensity ( $80 \%$ ) but there was no significant difference.

When the vibration stimulation was applied to Achilles tendon, there was a difference in the 
increase in muscle strength among soleus muscle, lateral gastrocnemius muscle, medial gastrocnemius muscle and tibialis anterior muscle depending on the perception threshold intensity and the sub-perception threshold intensity $(80 \%)$ but there was no significant difference.

Table 6. Muscle strength increasing rate [\%] of MVC in accordance with the vibration intensity applied to the tibialis anterior and Achilles tendon

\begin{tabular}{|c|c|c|c|c|c|c|}
\hline & & & Soleus & $\begin{array}{c}\text { Lateral } \\
\text { Gastrocnemius }\end{array}$ & $\begin{array}{c}\text { Medial } \\
\text { Gastrocnemius }\end{array}$ & $\begin{array}{l}\text { Tibialis } \\
\text { Anterior }\end{array}$ \\
\hline \multirow{6}{*}{$\begin{array}{l}\text { Tibialis } \\
\text { Anterior } \\
\text { Tendon }\end{array}$} & \multirow{2}{*}{$180 \mathrm{~Hz}$} & $100 \%$ & $7.1 \pm 3.6$ & $12.0 \pm 6.0$ & $12.2 \pm 6.1$ & $13.2 \pm 6.6$ \\
\hline & & $80 \%$ & $9.1 \pm 10.7$ & $9.9 \pm 7.8$ & $11.1 \pm 7.1$ & $8.9 \pm 6.5$ \\
\hline & \multirow{2}{*}{$190 \mathrm{~Hz}$} & $100 \%$ & $11.8 \pm 5.9$ & $13.1 \pm 6.5$ & $10.0 \pm 5.0$ & $12.7 \pm 6.4$ \\
\hline & & $80 \%$ & $6.2 \pm 13.0$ & $8.2 \pm 14.6$ & $5.7 \pm 8.5$ & $6.3 \pm 6.0$ \\
\hline & \multirow{2}{*}{$250 \mathrm{~Hz}$} & $100 \%$ & $12.5 \pm 6.2$ & $13.7 \pm 6.8$ & $10.1 \pm 5.1$ & $9.2 \pm 4.6$ \\
\hline & & $80 \%$ & $5.8 \pm 20.2$ & $15.2 \pm 19.5$ & $7.6 \pm 8.2$ & $3.1 \pm 6.9$ \\
\hline \multirow{6}{*}{$\begin{array}{l}\text { Achilles } \\
\text { Tendon }\end{array}$} & \multirow{2}{*}{$180 \mathrm{~Hz}$} & $100 \%$ & $16.0 \pm 8.0$ & $15.4 \pm 7.7$ & $16.3 \pm 8.1$ & $14.3 \pm 7.2$ \\
\hline & & $80 \%$ & $6.6 \pm 12.5$ & $13.1 \pm 13.0$ & $10.4 \pm 8.4$ & $20.2 \pm 17.9$ \\
\hline & \multirow{2}{*}{$190 \mathrm{~Hz}$} & $100 \%$ & $17.2 \pm 8.6$ & $12.4 \pm 6.2$ & $18.3 \pm 9.2$ & $18.0 \pm 9.0$ \\
\hline & & $80 \%$ & $10.9 \pm 11.6$ & $17.8 \pm 15.0$ & $14.3 \pm 10.4$ & $15.8 \pm 17.6$ \\
\hline & \multirow{2}{*}{$250 \mathrm{~Hz}$} & $100 \%$ & $8.4 \pm 4.2$ & $15.6 \pm 7.8$ & $14.7 \pm 7.3$ & $9.9 \pm 5.0$ \\
\hline & & $80 \%$ & $14.8 \pm 9.4$ & $13.7 \pm 19.9$ & $13.5 \pm 9.9$ & $18.7 \pm 11.4$ \\
\hline
\end{tabular}

Fig. 1 showed that if the vibration stimulation is applied to tibialis anterior tendon and Achilles tendon, the rate in the increase of muscle strength was statistically higher than that under non-stimulation. Based on these results, we can infer that the application of vibration stimulation to the muscle in the lower leg through the tibialis anterior tendon and Achilles tendon would affect the increase in muscle strength, thus giving a more positive effect on postural stability than under non-stimulation. In addition, it was found out that the rate of increase in muscle strength was higher in Achilles tendon than that in tibialis anterior tendon. This shows that the Achilles tendon is higher in terms of the increase in muscle strength due to vibration stimulation. Table 5 showed that when vibration stimulation is applied to tibialis anterior tendon and Achilles tendon, the rates in the increase of muscle strength for soleus muscle, lateral gastrocnemius muscle, medial gastrocnemius muscle and tibialis anterior muscle were the highest at the frequency of $180 \mathrm{~Hz}$. This indicates that vibration frequency has an effect on the increase in muscle strength. Table 6 showed that there was a difference in the increase in muscle strength depending on the vibration stimulation intensity but it was not significant. This means that the stimulation in the perception threshold and sub-perception threshold has an effect on the increase in muscle strength.

\subsection{Change in MRT depending on the condition of vibration stimulation}

The change in muscle reaction time depending on the conditions of vibration stimulation was analyzed based on MRT data under the condition of non-stimulation.

Fig. 2 shows that muscle reaction time was more reduced in vibration stimulation than in non-stimulation. It was found out that when vibration stimulation is applied to tibialis anterior tendon, the muscle reaction time for soleus muscle, lateral gastrocnemius muscle, medial gastrocnemius muscle and tibialis anterior muscle was more significantly reduced than under non-stimulation (Soleus $p$-value $=0.005$, Lateral Gastrocnemius $p$-value $=0.013$, Medial Gastrocnemius $p$-value $=0.005$, Tibialis Anterior $p$-value $=0.012)$. It was also found out that when vibration stimulation is applied to Achilles tendon, muscle reaction time for soleus muscle, lateral gastrocnemius muscle, medial gastrocnemius muscle and tibialis anterior muscle was more significantly reduced than under non-stimulation (Soleus $p$-value $=0.005$, Lateral Gastrocnemius $p$-value $=0.009$, Medial Gastrocnemius $p$-value $=0.005$, Tibialis Anterior $p$-value $=0.009$ )

It was found (Table 7) out that when vibration stimulation is applied to tibialis anterior tendon, muscle reaction time at $180 \mathrm{~Hz}$ was more significantly reduced than under non-stimulation and in 
a faster way than those at $180 \mathrm{~Hz}$ and $250 \mathrm{~Hz}$. It was also found out that when vibration stimulation is applied to Achilles tendon, muscle reaction time at $180 \mathrm{~Hz}$ was more significantly reduced than under non-stimulation and in a faster way than those at $180 \mathrm{~Hz}$ and $250 \mathrm{~Hz}$.

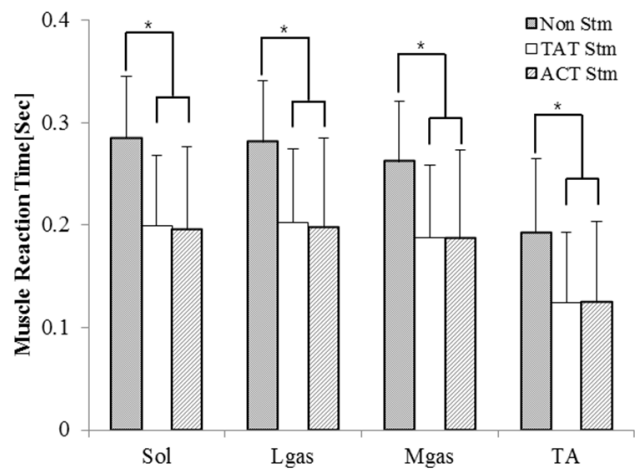

Fig. 2. Change in MRT under vibration stimulation applied to the Achilles tendon and tibialis anterior tendon and under non-stimulation (sec). TAT Stm: Tibialis Anterior Tendon Stimulation, ACT Stm: Achilles Tendon Stimulation. *: $p<0.05$. Non-Stimulation versus Stimulation

Table 7. Change in MRT depending on the frequency in vibration stimulation applied to the tibialis anterior tendon and Achilles tendon $(\mathrm{sec})$

\begin{tabular}{|c|c|c|c|c|c|}
\hline & & Soleus & $\begin{array}{c}\text { Lateral } \\
\text { Gastrocnemius }\end{array}$ & $\begin{array}{c}\text { Medial } \\
\text { Gastrocnemius }\end{array}$ & $\begin{array}{c}\text { Tibialis } \\
\text { Anterior }\end{array}$ \\
\hline None & & $0.285 \pm 0.030^{\mathrm{a}}$ & $0.281 \pm 0.030^{\mathrm{a}}$ & $0.262 \pm 0.029^{\mathrm{a}}$ & $0.193 \pm 0.036^{\mathrm{a}}$ \\
\hline Tibialis & $180 \mathrm{~Hz}$ & $0.171 \pm 0.033^{\mathrm{a}}$ & $0.181 \pm 0.036^{\mathrm{a}}$ & $0.170 \pm 0.036^{\mathrm{a}}$ & $0.124 \pm 0.036^{\mathrm{a}}$ \\
\cline { 2 - 6 } Anterior & $190 \mathrm{~Hz}$ & $0.188 \pm 0.037^{\mathrm{a}}$ & $0.190 \pm 0.045^{\mathrm{a}}$ & $0.188 \pm 0.039^{\mathrm{a}}$ & $0.128 \pm 0.049^{\mathrm{a}}$ \\
\cline { 2 - 6 } Tendon & $250 \mathrm{~Hz}$ & $0.191 \pm 0.041^{\mathrm{a}}$ & $0.195 \pm 0.043^{\mathrm{a}}$ & $0.178 \pm 0.043^{\mathrm{a}}$ & $0.128 \pm 0.040^{\mathrm{a}}$ \\
\hline \multirow{2}{*}{$\begin{array}{c}\text { Achilles } \\
\text { Tendon }\end{array}$} & $180 \mathrm{~Hz}$ & $0.174 \pm 0.038^{\mathrm{a}, \mathrm{b}}$ & $0.182 \pm 0.038^{\mathrm{a}} \mathrm{b}$ & $0.168 \pm 0.037^{\mathrm{a}, \mathrm{b}}$ & $0.124 \pm 0.029^{\mathrm{a}}$ \\
\cline { 2 - 6 } & $190 \mathrm{~Hz}$ & $0.201 \pm 0.026^{\mathrm{a}}$ & $0.205 \pm 0.031^{\mathrm{a}, \mathrm{b}}$ & $0.197 \pm 0.029^{\mathrm{a}, \mathrm{b}}$ & $0.122 \pm 0.031^{\mathrm{a}}$ \\
\cline { 2 - 6 } & $250 \mathrm{~Hz}$ & $0.222 \pm 0.035^{\mathrm{a}, \mathrm{b}}$ & $0.226 \pm 0.039^{\mathrm{a}, \mathrm{b}}$ & $0.207 \pm 0.035^{\mathrm{a}, \mathrm{b}}$ & $0.128 \pm 0.043^{\mathrm{a}}$ \\
\hline a $: P<0.05$. Non-Stimulation versus other frequencies, ${ }^{\mathrm{b}}: p<0.05 .180 \mathrm{~Hz}$ versus other frequencies \\
\hline
\end{tabular}

Table 8 When the vibration stimulation is applied to tibialis anterior tendon, there was a difference in muscle reaction time for soleus muscle, lateral gastrocnemius muscle, medial gastrocnemius muscle and tibialis anterior muscle between in perception threshold intensity and sub-perception threshold intensity but there was no significant difference. When the vibration stimulation is applied to Achilles tendon, there was a difference in muscle response time for soleus muscle, lateral gastrocnemius muscle, medial gastrocnemius muscle and tibialis anterior muscle between in perception threshold intensity and sub-perception threshold intensity but there was no significant difference.

Fig. 2 shows that if the vibration stimulation is applied to tibialis anterior tendon and Achilles tendon, the muscle reaction time was further reduced than under non-stimulation, thus showing a significant difference. The results infer that there would be reduction in time required to transmit and process the information passing through the route of sensory system - central neural system - cooperation between neuron and muscle and the muscular system. Table 7 showed that when vibration stimulation is applied to tibialis anterior tendon and Achilles tendon, muscle reaction time was the fastest in the frequency of $180 \mathrm{~Hz}$ for soleus muscle, lateral gastrocnemius muscle, medial gastrocnemius muscle and tibialis anterior muscle. From this, we can understand that muscle reaction time is affected by vibration frequency. Table 8 showed that a different muscle reaction time appeared depending on the strength of vibration stimulation with no significant difference. This means that the stimulation in the perception threshold intensity and sub-perception threshold intensity has an effect on the reduction of muscle reaction time. 
Table 8. Change in MRT depending on the intensity in vibration stimulation applied to the tibialis anterior tendon and Achilles tendon ( $\mathrm{sec})$

\begin{tabular}{|c|c|c|c|c|c|c|}
\hline & & & Soleus & $\begin{array}{c}\text { Lateral } \\
\text { Gastrocnemius }\end{array}$ & $\begin{array}{c}\text { Medial } \\
\text { Gastrocnemius }\end{array}$ & $\begin{array}{c}\text { Tibialis } \\
\text { Anterior }\end{array}$ \\
\hline \multirow{6}{*}{$\begin{array}{l}\text { Tibialis } \\
\text { Anterior } \\
\text { Tendon }\end{array}$} & \multirow{2}{*}{$180 \mathrm{~Hz}$} & $100 \%$ & $0.171 \pm 0.033$ & $0.181 \pm 0.036$ & $0.170 \pm 0.036$ & $0.124 \pm 0.036$ \\
\hline & & $80 \%$ & $0.204 \pm 0.051$ & $0.203 \pm 0.052$ & $0.189 \pm 0.054$ & $0.107 \pm 0.028$ \\
\hline & \multirow{2}{*}{$190 \mathrm{~Hz}$} & $100 \%$ & $0.188 \pm 0.037$ & $0.190 \pm 0.045$ & $0.188 \pm 0.039$ & $0.128 \pm 0.049$ \\
\hline & & $80 \%$ & $0.191 \pm 0.043$ & $0.197 \pm 0.046$ & $0.191 \pm 0.044$ & $0.118 \pm 0.038$ \\
\hline & \multirow{2}{*}{$250 \mathrm{~Hz}$} & $100 \%$ & $0.191 \pm 0.041$ & $0.195 \pm 0.043$ & $0.178 \pm 0.043$ & $0.128 \pm 0.040$ \\
\hline & & $80 \%$ & $0.203 \pm 0.039$ & $0.207 \pm 0.039$ & $0.188 \pm 0.045$ & $0.143 \pm 0.047$ \\
\hline \multirow{6}{*}{$\begin{array}{l}\text { Achilles } \\
\text { Tendon }\end{array}$} & \multirow{2}{*}{$180 \mathrm{~Hz}$} & $100 \%$ & $0.174 \pm 0.038$ & $0.182 \pm 0.038$ & $0.168 \pm 0.037$ & $0.124 \pm 0.029$ \\
\hline & & $80 \%$ & $0.191 \pm 0.038$ & $0.194 \pm 0.041$ & $0.182 \pm 0.039$ & $0.124 \pm 0.037$ \\
\hline & \multirow{2}{*}{$190 \mathrm{~Hz}$} & $100 \%$ & $0.201 \pm 0.026$ & $0.205 \pm 0.031$ & $0.197 \pm 0.029$ & $0.122 \pm 0.031$ \\
\hline & & $80 \%$ & $0.208 \pm 0.036$ & $0.210 \pm 0.034$ & $0.191 \pm 0.035$ & $0.115 \pm 0.033$ \\
\hline & \multirow{2}{*}{$250 \mathrm{~Hz}$} & $100 \%$ & $0.222 \pm 0.035$ & $0.226 \pm 0.039$ & $0.207 \pm 0.035$ & $0.128 \pm 0.043$ \\
\hline & & $80 \%$ & $0.201 \pm 0.033$ & $0.201 \pm 0.034$ & $0.180 \pm 0.038$ & $0.132 \pm 0.034$ \\
\hline
\end{tabular}

\section{Conclusions}

This study was conducted on the stimulation intensity which is less than the perception threshold which the human body can feel based on SEPs. The findings are as follows. It was found that when the stimulation which has the intensity of 70-95\% of the sub-perception threshold to tibialis anterior tendon and Achilles tendon, the change in the SEPs was observed at $80 \%$ of the perception threshold. So, it was concluded that the vibration stimulation which is less than the perception threshold generates SEPs, thus making a significant effect on the human body. In addition, a research was made on how vibration stimulation applied to tibialis anterior tendon and Achilles tendon affect the triceps surae muscle and tibialis anterior muscle depending on the conditions of vibration stimulation (existence of stimulation, frequency and intensity). The findings are as follows. When vibration stimulation was applied, muscle strength increased and muscle reaction time decreased compared to non-stimulation. Muscle strength and muscle reaction time were the best when the frequency was at $180 \mathrm{~Hz}$. Even under vibration stimulation intensity of sub-perception threshold, there are the effects such as an increase in muscle strength and reduction in muscle reaction time. If these findings are applied to the people whose postural stabilities have been lowered due to aging and accident, the stimulation would have a positive effect on the improvement in the risk of abnormal gait and falls.

\section{Acknowledgements}

This work was supported by the National Research Foundation of Korea (NRF) Grant funded by the Korea Government (MSIP) (NRF-2014R1A2A1A11053073).

\section{References}

[1] Shumway A., Woollacott M. H. Motor Control: Theory and Practical Applications. Lippincott Williams and Wilkins Inc., Balltimore, 2007.

[2] Maurer C., Mergner T., Bolha B., Hlavacka F. Vestibular, visual, and somatosensory contributions to human control of upright stance. Neuroscience Letter, Vol. 281, Issues 2-3, 2000, p. 99-102.

[3] Handrigan G., Hue O., Simoneau M., Corbeil P., Marceau P., Marceau S. Weight loss and muscular strength affect static balance control. International Journal of Obesity, Vol. 34, Issue 5, 2010, p. $936-942$.

[4] King G. W., Stylianou A. P., Kluding P. M., Jernigan S. D., Luchies C. W. Effect of age and localized muscle fatigue on ankle plantar flexor torque development. Journal of Geriatric Physical Therapy, Vol. 35, Issue 1, 2012, p. 8-14.

[5] Guyton A. C., Hall J. E. Medical Physiology. 10th Edition, W.B. Saunders Company, Philadelphia, 2000. 
[6] Gravelle D. C., Laughton C. A., Dhruv N. T., Datdare K. D., Niemi J. B., Lipsitz L. A., Collins J. J. Noise-enhanced balance control in older adults. Neuroreport, Vol. 13, Issue 15, 2002, p. 1853-1856.

[7] Dickstein R., Laufer Y., Katz M. TENS to the posterior aspect of the legs decreases postural sway during stance. Neuroscience Letters, Vol. 393, Issue 1, 2006, p. 51-55.

[8] Lekhel H., Popov K. E., Anastassopoulos D., Bronstein A., Bhatia K., Marsdem C. D., Gresty M. Postural responses to vibration of neck muscles in patients with idiopathic Torticollis. Journal of Neurology, Vol. 120, Issue 4, 1997, p. 583-591.

[9] Priplata A., Niemi J. Salen M., Harry J. Lipsitz L. A., Collins J. J. Noise-enhance human balance control. Physical Review Letters, Vol. 89, Issue 23, 2002, p. 1-4.

[10] Cruccu G., Aminoff M. J., Curio G., Guerit J. M., Kakigi R., Mauguiere F., Rossini P. M., Treede R. D., Garcia-Larrea L. Recommendations for the clinical use of somatosensory-evoked potentials. Clinical Neurophysiology, Vol. 119, Issue 8, 2008, p. 1705-1719.

[11] Ara J., Hwang S. H., Song T., Khang G. Electrically-elicited tactile sensation for different modulation types, polarities and waveforms of stimulation pulse trains. International Journal of Precision Engineering and Manufacturing, Vol. 13, Issue 10, 2012, p. 1911-1916.

[12] Kwak K. Y., Kim H. G., Kim D. W. Variation of ankle biomechanical property according to vibro-perception threshold and vibration frequency. Biomedical Engineering Letters, Vol. 6, Issue 1, 2016, p. 16-25.

[13] Merle L. Fox's Physiological Vasis for Exercise and Sport. 6th Edition, WCB McGraw-Hill, Boston, 1998.

[14] Kim J. W., Jeong H. Y., Kim H. H., Kwon Y. R., Eom G. M., Lee J. W., Kim K. S., Jun J. H., Kwon T. K. Comparison of reaction times of ankle joint muscles in the elderly men and women. Journal of Biomedical Engineering Research, Vol. 33, Issue 4, 2012, p. 163-168.

[15] Hiroki N., Misaki O., Mari N., Manabu S. Effects of aerobic exercise under different thermal conditions on human somatosensory processing. American Journal of Physiology, Vol. 311, Issue 4, 2016, p. 629-636.

[16] Reinacher M., Becker R., Villringer A., Ritter P. Oscillatory brain states interact with late cognitive components of the somatosensory evoked potential. Journal of Neuroscience Methods, Vol. 183, Issue 1, 2009, p. 49-56.

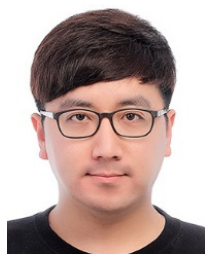

Huigyun Kim is now with the Department of Healthcare Engineering, Chonbuk National University, Jeonju-si, Jeollabuk-do, Republic of Korea. His current research interests include neuronal electrophysiology and sensory-motor integration.

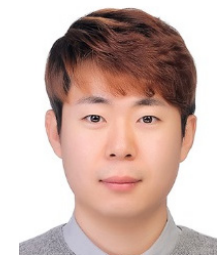

Kiyoung Kwak is now with the Department of Healthcare Engineering, Chonbuk National University, Jeonju-si, Jeollabuk-do, Republic of Korea. His current research interests include neuro-musculoskeletal biomechanics and somatosensory-motor integration.

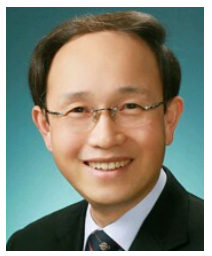

Dongwook Kim received Ph.D. degree in Biomedical Engineering from Hokkaido University, Sapporo, Japan in 1995. Now he is a Professor at Department of Biomedical Engineering, Director of Educational Development Institute and Advancement of College Education in Chonbuk National University, Jeonju-si, Jeollabuk-do, Republic of Korea. His current research interests include biomedical engineering, rehabilitation engineering, sensory-motor integration, diagnosis and healthcare system. 